\title{
MÉTODO DE IMPLEMENTAÇÃO DE SISTEMA DE DIAGNÓSTICO DE FALTA PARA SUBESTAÇÕES BASEADO EM REDES DE PETRI
}

\author{
Raimundo Furtado Sampaio* \\ rfurtado@coelce.com.br
}

Giovanni Cordeiro Barroso ${ }^{\dagger}$

gcbefisica.ufc.br

\author{
Ruth Pastôra Saraiva Leão \\ rleao@dee.ufc.br \\ *COMPANHIA ENERGÉTICA DO CEARÁ \\ †DEPARTAMENTO DE FÍSICA-UNIVERSIDADE FEDERAL DO CEARÁ \\ ‡DEPARTAMENTO DE ENGENHARIA ELÉTRICA-UNIVERSIDADE FEDERAL DO CEARÁ
}

\section{ABSTRACT}

This paper presents a methodology for the development of a Fault Diagnosis System (FDS) based on Hierarchical Colored Petri Nets. The proposed FDS aims to evaluate the information from the SCADA and to present a fault diagnosis sucint and accurate to the power system operators, causing the task of fault diagnosis faster, reliable and less stressful. The proposed methodology is developed and applied to a typical distribution substation with digital relays, 15 MVA, $69 \mathrm{kV} / 13,8 \mathrm{kV}$, owned by the local distribution utility, Companhia Energética do Ceará (Brazil).

KEYWORDS: Substation automation, fault diagnosis, colored Petri nets, SCADA system.

\section{RESUMO}

Este trabalho apresenta um método de desenvolvimento de Sistema de Diagnóstico de Faltas (SDF) utilizando Redes de Petri Coloridas Hierárquicas. O SDF proposto visa analisar as informações advindas do SCADA e apresentar diagnóstico

\footnotetext{
Artigo submetido em 22/06/04

1a. Revisão em 19/10/04

2a. Revisão em 27/04/05

3a. Revisão em 17/06/05

4a. Revisão em 21/12/05

Aceito sob recomendação do Ed. Assoc. Prof. Paulo Eigi Miyagi
}

de falta sucinto e preciso aos operadores de sistema elétrico, tornando a tarefa de diagnóstico de falta, mais rápida, confiável e menos estressante. O método proposto é desenvolvido e aplicado a uma subestação típica de distribuição, digitalizada, de 15 MVA, 69/13,8 kV, pertencente ao sistema de distribuição da Companhia Energética do Ceará - COELCE.

PALAVRAS-CHAVE: Automação de subestação, diagnóstico de faltas, redes de Petri coloridas, sistema SCADA.

\section{INTRODUÇÃO}

A crescente utilização de sofisticados sistemas de informação para supervisão e controle dos sistemas elétricos, com aplicação de relés multifunção baseados em microprocessadores, e sistemas SCADA distribuídos, tem contribuído para o aumento da segurança e da confiabilidade dos sistemas elétricos. No entanto, para os profissionais da operação, a tarefa de análise e diagnósticos de faltas tornou-se complexa. Após uma ocorrência nos modernos sistemas elétricos, as faltas são analisadas com base nas informações disponibilizadas pelos sistemas SCADA. Neste processo, o profissional deve analisar e identificar as possíveis causas da falta, e tomar a decisão de restabelecer o sistema elétrico com segurança e em tempo hábil. Apesar de comum, esta é uma tarefa estressante e complexa que pode demandar longo tempo em caso de faltas envolvendo falha no sistema de proteção, descoordenação 
de proteção, ou múltiplas faltas no sistema elétrico.

O sistema SCADA de subestação coleta eventos e alarmes detalhados referentes a manobras, alarmes de superação de limites, sinalizações, ordens de comando, partidas e atuações por fase e neutro da proteção, abertura e fechamento de equipamento de disjunção, rearmes, valores analógicos de tensão e de corrente de equipamentos envolvidos na falta, todos com seus respectivos registros de tempo. Para tomar a decisão de repor o sistema elétrico, o operador necessita analisar apenas os dados referentes aos equipamentos de disjunção e funções de proteção que atuaram e/ou falharam. No entanto, a massa de dados disponibilizados pelo SCADA dificulta a análise por parte do profissional de operação, podendo leválo a erros de interpretação ou retardar o restabelecimento do sistema. Existe, portanto, uma demanda por uma ferramenta computacional efetivamente eficiente, capaz de analisar as informações provenientes do SCADA com rapidez e exatidão.

O sistema de diagnóstico de faltas é uma ferramenta de suporte ao operador para identificação, localização e diagnóstico de faltas simples e complexas, auxiliando a área de operação das empresas de energia durante condições que demandam rapidez e precisão na tomada de decisão.

Este artigo tem por objetivo apresentar um método de implementação de Sistemas de Diagnóstico de Faltas (SDF) para subestação baseado em Redes de Petri Coloridas Hierárquicas (RPC-H) (Sampaio et alii, Junho 2002; Sampaio et alii, 2003). Um modelo de SDF foi desenvolvido para a subestação de distribuição da Companhia Energética do Ceará.

O artigo está organizado em seis seções. Na introdução é apresentada a motivação para o desenvolvimento de uma ferramenta computacional de auxílio ao operador voltada ao diagnóstico de falta em tempo real. Na seção dois são apresentadas as técnicas utilizadas no desenvolvimento de funções avançadas de apoio à tomada de decisão, e as vantagens das redes de Petri na modelagem de sistemas de diagnóstico de faltas. A seção três apresenta o conceito de sistema centralizado e distribuído e a seção quatro descreve o método aplicado no desenvolvimento do SDF. Na seção cinco o método baseado em redes de Petri coloridas hierárquicas é usado para modelar o SDF do vão de banco de capacitores. Finalmente as conclusões são apresentadas na seção seis.

\section{TÉCNICAS COMPUTACIONAIS DE APOIO À TOMADA DE DECISÃO}

Ao longo dos anos vários trabalhos têm sido propostos utilizando técnicas computacionais modernas para implementação de ferramentas avançadas de automação para auxiliar os profissionais da operação de sistemas elétricos no processo de tomada de decisão. Dentre as ferramentas propostas têmse os Sistemas de Tratamento de Alarmes, os Sistemas de Diagnóstico de Faltas e os Sistemas de Reposição Automáticos.

Para implementação desses sistemas têm-se utilizado técnicas computacionais como redes neurais, lógica fuzzy, sistemas baseados em conhecimento, diagrama de causa e efeito, e redes de Petri (Vale et alii, 2003; Infra et alli, 1995; Brittes, 1998; Silva et alli, 1995; Lee et alli, 2000; Chen et alli, 2000; Yang and Yokohama, 1995; Lo et alli, 1997; Sampaio et alli, Junho 2002, Set. 2002, 2003).

Dentre as várias técnicas mencionadas, as mais utilizadas são sistemas especialistas e redes neurais. Os sistemas especialistas oferecem soluções ao diagnóstico de faltas, mas esta técnica apresenta dificuldade de manutenção e/ou de adequação da base de conhecimento, devido ao enorme número de regras, o que pode facilmente gerar inconsistências. Ademais, o tempo de resposta normalmente não é satisfatório a uma aplicação em tempo real devido à representação de conhecimento e o mecanismo de inferência (Chen et alli, 2000).

O uso de rede neural artificial é uma solução potencial para os problemas encontrados com os sistemas especialistas. Porém alguns problemas com as redes neurais ainda permanecem sem solução em aplicações práticas como convergência lenta no processo de treinamento, e a determinação dos parâmetros de rede como de unidades escondidas, camadas, e taxa de aprendizagem (Chen et alli, 2000). Além disso, nas redes neurais não se pode determinar como os resultados são obtidos, ou como o diagnóstico é alcançado a partir do resultado, e quando a configuração do sistema em estudo muda, a rede neural necessita ser treinada novamente.

As Redes de Petri (RP) são uma ferramenta matemática com visualização gráfica, capaz de modelar, analisar, controlar, validar e implementar diferentes sistemas, em especial sistemas a eventos discretos (Murata, 1989). As RPs têm sido aplicadas na modelagem, análise e implementação de sistemas de controle, restauração, e diagnóstico de falta em sistemas de potência (Yang and Yokohama, 1995; Lo et alli, 1997; Sampaio et alli, Junho 2002, Set. 2002, 2003).

Durante as últimas décadas, muitos sistemas computacionais desenvolvidos para monitoramento e controle de sistemas de potência em tempo real não foram concebidos com capacidade de expansibilidade e manutenibilidade, tornando-se obsoletos mediante a necessidade de atualização dos softwares para atender aos novos requerimentos operacionais dos sistemas de potência (Zhu, 1997).

O método proposto neste trabalho baseado em uma extensão das RPs apresenta vantagens em relação às demais técnicas computacionais. No SDF proposto, modelos de rede de Pe- 
tri são desenvolvidos para o sistema de proteção de cada vão da subestação, os quais podem ser validados enquanto modelos e posteriormente implementados. A modularidade do sistema proposto torna a tarefa de manutenção e modificação do sistema de diagnóstico fácil e rápida. Por serem modulares, os modelos podem também ser reutilizados e facilmente adaptados para outro vão da subestação ou para subestações de diferentes topologias. Além disso, as atuações de relés e aberturas de disjuntores podem ser inferidas mais corretamente utilizando esta técnica, dado que as RPs permitem:

a) Representar através dos modelos desenvolvidos as causalidades entre faltas nos componentes do sistema de potência, ações de relés de proteção e disjuntores;

b) Representar explicitamente os estados de operação dos dispositivos de proteção (atuação correta/falsa de relé e defeito em disjuntor);

c) Manusear informações de seqüência de operação de disjuntores e relés;

d) Permitir a validação dos modelos independente da dinâmica do sistema de potência;

e) Assegurar que um determinado modelo de SDF possua a qualidade desejada;

f) Possível redução do tempo de inferência, por serem as RPs adequadas ao processamento paralelo;

g) Permitir comparação entre diferentes modelos de proteção.

Adicionalmente, o processo de diagnóstico baseado em RPs apresenta a vantagem de empregar modelos matriciais, de fácil manipulação e de baixo custo computacional, o que reduz o tempo de processamento e aumenta a exatidão dos resultados em relação aos complexos processos de pesquisa normalmente utilizados em outras técnicas computacionais, especialmente quando o grau de complexidade do sistema cresce. Além do mais, o método proposto pode também esclarecer incertezas nos sinais recebidos e possui maior capacidade de adaptação às mudanças na configuração da rede.

\subsection{Redes de Petri Coloridas Hierárqui- cas}

Neste trabalho foi utilizada uma extensão de RP denominada de Redes de Petri Coloridas Hierárquicas (RPC-H). As RPC$\mathrm{H}$ são capazes de representar modelos com alto nível de abstração, melhor representação gráfica, maior compactação, e dados estruturados que permitem a construção modular da rede de forma similar às usadas em linguagens de programação estruturada. A capacidade de uma RPC-H modelar um sistema hierarquicamente resulta do fato de que uma rede inteira pode ser substituída por um simples lugar ou transição, em um nível mais abstrato e compacto, denominados, respectivamente, de lugar de fusão e transição de substituição. Assim, lugares e transições podem ser substituídos por subredes para prover um modelo mais detalhado (Jansen, 1992, 1994, 1997).

O modelo RPC-H, através de suas fichas coloridas, explicita todas as funções e estados dos elementos de um sistema de proteção. Assim, o estado inicial da RPC é representado através dos eventos disponibilizados pelo SCADA relacionados aos dispositivos e equipamentos do sistema de proteção que atuaram durante uma falta. A RPC é então executada e seu estado final representará com precisão o vão em que ocorreu a falta e/ou falha, a função de proteção que atuou com indicação da fase, o disjuntor que interrompeu a falta, e as prováveis causas da ocorrência, diminuindo assim, o tempo de análise da falta e de tomada de decisão para reposição do sistema.

Para a análise de falta em uma subestação em um procedimento convencional, o operador deve identificar na lista completa de eventos disponibilizada pelo sistema supervisório SCADA os equipamentos que atuaram e/ou falharam. De posse destes dados, o operador deve identificar a partir de uma análise o local e a causa da falta para tomar a decisão de repor o sistema ou chamar a equipe de manutenção para a solução do problema. No processo de diagnóstico automático, após a falta, o modelo do SDF, baseado em RPC, recebe os dados disponibilizados na lista de eventos do SCADA, seleciona os dados de interesse usados para a marcação inicial da RPC, executa a rede e apresenta as funções de proteção que atuaram e/ou falharam, a área em que ocorreu a falta e as causas primárias da mesma. Sendo assim, o modelo RPC proposto é um modelo não cíclico, cuja marcação inicial é definida somente pelos equipamentos envolvidos numa falta específica, seja ela simples ou múltipla, e a marcação final determina quais equipamentos atuaram e/ou falharam, definindo, assim, uma ou mais áreas específicas de ocorrência da falta. Após a análise e armazenamento da marcação final (diagnóstico), o sistema é reiniciado, ou seja, retorna ao estado de espera de uma nova marcação inicial quando da ocorrência de outra falta, descartando a marcação final anteriormente encontrada.

Para análise lógica dos modelos, inicialmente foram, construídos modelos cíclicos e pode-se constatar que ao utilizar uma marcação inicial com todas as possíveis funções de relés e todos os disjuntores associados a uma dada zona de proteção, a RPC era viva, limitada e reversível. No entanto, para a aplicação do SDF, uma rede cíclica não apresentaria com rapidez um estado específico do sistema que é o estado após a ocorrência de uma falta. Desta forma, foi construída uma 

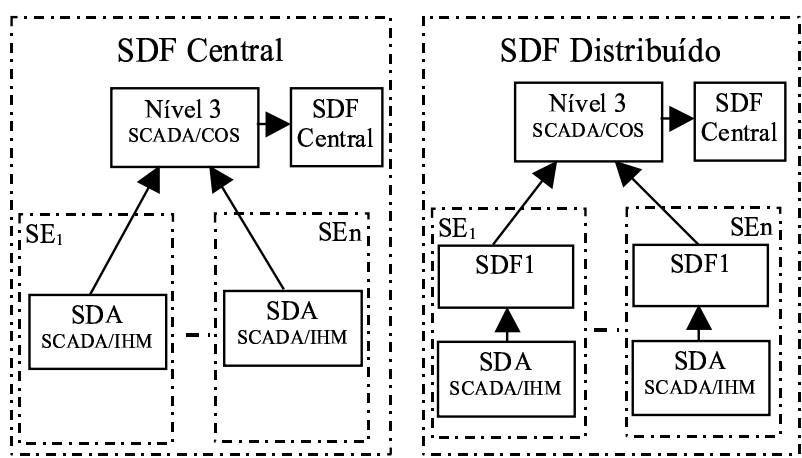

Figura 1: Configuração de SDF Centralizado (a) e SDF Distribuído (b)

rede não cíclica que a cada execução apresenta somente uma instanciação das variáveis e, por conseguinte, o conjunto de estados é pequeno e rápido se ser alcançado.

\section{ABORDAGENS PARA DESENVOLVI- MENTO DE UM SDF}

Existem basicamente duas abordagens que podem ser adotadas para o desenvolvimento de um SDF, a abordagem centralizada e a distribuída, conforme ilustrado na Figura 1.

$\mathrm{Na}$ abordagem centralizada, Figura 1 (a), o SDF é integrado a um sistema SCADA/DMS no Centro de Operação do Sistema (COS), realizando o diagnóstico a partir das informações reportadas para este sistema. Na abordagem distribuída, ilustrada na Figura 1 (b), os SDF são integrados aos sistemas SCADA/SDA (Sistemas Digitais de Automação) das subestações (SE) e reportam os resultados dos diagnósticos ao COS.

O SDF distribuído apresenta dentre outras as seguintes vantagens sobre o SDF centralizado: a) utiliza para análise apenas os dados disponíveis na subestação, otimizando e reduzindo a dimensão do sistema; b) apresenta maior confiabilidade, tendo em vista o resultado do diagnóstico não estar tão dependente do meio de comunicação; c) dispõe de informações importantes para o diagnóstico de faltas que comumente não são reportadas para o COS, a exemplo dos dados de partida de relé; e d) libera os canais de comunicação para outros fins uma vez que apenas o resultado do diagnóstico é transferido ao nível superior.

No método distribuído, os SDF locais realizam os diagnósticos das faltas a partir dos eventos reportados pelos respectivos SCADA da subestação. Os SDF geram relatórios locais às subestações, bem como disponibilizam ao COS os resultados de diagnósticos de faltas. No COS, os dados de diagnósticos de faltas são disponibilizados para os operadores e

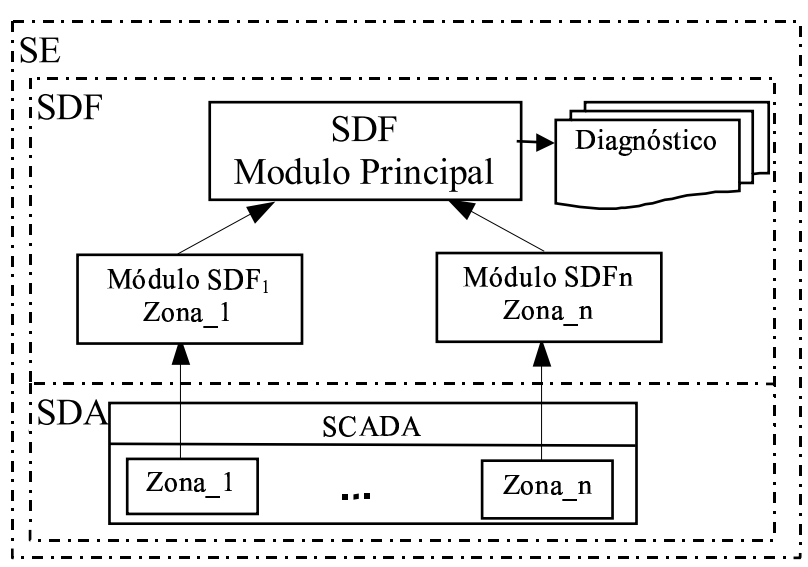

Figura 2: Estrutura Modular de um SDF para Subestação.

reportados para um SDF central responsável pelos diagnósticos de faltas que envolvem subestações interligadas.

\section{MÉTODO PROPOSTO PARA DESEN- VOLVIMENTO DE UM SDF}

O método desenvolvido para sistemas de diagnóstico de faltas apresentada neste trabalho utiliza a abordagem distribuída e está baseada no particionamento da subestação por zona de proteção caracterizando o SDF como localmente distribuído.

O Sistema de Diagnóstico de Falta proposto, conforme ilustrado na Figura 2, é integrado ao sistema SCADA da subestação e realiza os diagnósticos das faltas a partir de uma lista de eventos reportados pelo sistema SCADA da subestação.

Como pode ser observado o SDF é distribuído, ou seja, para cada zona de proteção um módulo SDF é construído e integrado ao módulo principal. O módulo principal é responsável pela integração de todos os sub-módulos e a partir dele obtêm-se diagnósticos de faltas que envolvem mais de uma zona de proteção. O módulo de cada zona possui interface com o sistema SCADA da subestação, recebendo os eventos das ocorrências na subestação. A partir destes eventos, cada módulo realiza diagnósticos de faltas referentes àquela zona de proteção específica. Desta forma, o método proposto permite a realização de diagnósticos de ocorrências no sistema elétrico com diferentes graus de complexidade, tais como: falta simples, relacionada a um único vão, ou faltas que envolvem mais de um vão da subestação tais como faltas múltiplas, descoordenação da proteção, e falhas no sistema de proteção.

As etapas de desenvolvimento do SDF podem ser assim resumidas:

- A subestação é dividida em zonas de proteção. 
- Uma RPC-H é desenvolvida com um módulo principal integrado aos módulos individuais de cada zona de proteção.

- Uma interface para integrar o SDF ao sistema SCADA é desenvolvida visando a coleta e a filtragem dos eventos relacionados a cada zona de proteção, os quais são identificados na base de dados do SCADA, decodificados e alocados como marcação inicial das respectivas redes.

- Desenvolvimento de interface para apresentação do resultado do diagnóstico ao operador.

Dada a ocorrência de um evento, o SDF através da interface com o SCADA coleta, filtra e codifica os dados disponibilizados pelo SCADA como marcação inicial para a RPC-H. De posse da marcação inicial, a rede é executada e seu estado final indica com precisão o vão onde ocorreu a falta, a função de proteção que operou, com indicação da(s) fase(s) envolvidas, e o disjuntor que interrompeu a falta. A marcação final da rede é convertida em relatório através de saída para apresentação dos resultados do diagnóstico.

Uma vez realizado o diagnóstico, armazenadas as informações relativas ao evento e apresentado o relatório, a RPC$\mathrm{H}$ que foi executada é levada ao seu estado inicial, ou seja, elimina-se a marcação final encontrada e a rede está novamente pronta para receber uma nova marcação inicial e ser executada novamente quando da ocorrência de outro evento.

\section{DESENVOLVIMENTO DE SDF BASE- ADO EM RPC-H}

Para aplicação do SDF foi selecionada a subestação distribuidora Beberibe (SE BBR) cujo diagrama unifilar simplificado de proteção é apresentado na Figura 3.

A filosofia de proteção para a SE BBR consiste na instalação de disjuntores entre cada dois vãos da subestação e relés associados aos vãos, de forma que zonas de proteção individuais são estabelecidas ao redor de cada vão. As zonas de proteção apresentam regiões de sobreposição, evitando a possibilidade de áreas não protegidas na subestação. Se uma falta ocorre nessas áreas sobrepostas, mais de um relé de proteção pode operar.

Seguindo o método proposto para o desenvolvimento do SDF, a SE BBR foi dividida nas seguintes zonas de proteção:

- Zona de proteção de alta tensão (AT): protegida através do relé Rel que atua sobre o disjuntor principal (12C5) e/ou de transferência (12D1), através de uma chave de transferência da proteção (43C5).

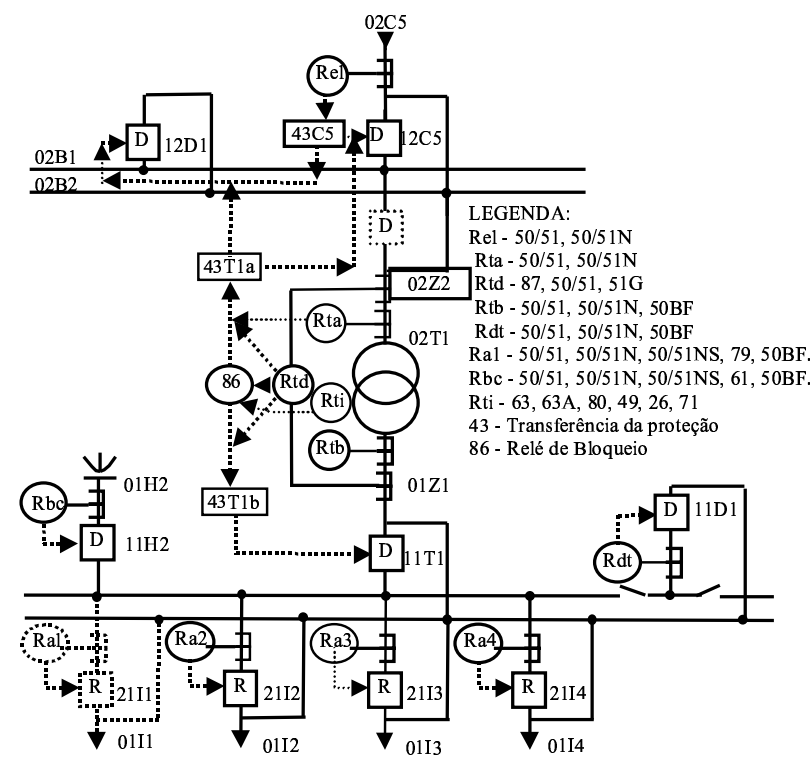

Figura 3: Diagrama Unifilar da Subestação Beberibe da Companhia Energética do Ceará.

- Zona de proteção do transformador (TR): protegida através dos relés Rta, Rtd e Rti que atuam sobre os disjuntores principais e de transferência de alta e/ou média tensão $(12 \mathrm{C} 5,12 \mathrm{D} 1,11 \mathrm{~T} 1,11 \mathrm{D} 1)$ através de chaves de transferência da proteção (43T1a e 43T1b).

- Zona de proteção de média tensão (MT): protegida através do relé Rtb associado ao disjuntor geral do barramento de média tensão (11T1) ou através do relé Rdt associado ao disjuntor de transferência (11D1). O disjuntor de transferência 11D1 pode substituir o disjuntor geral $11 \mathrm{~T} 1$ ou qualquer um dos religadores, para tanto o relé Rdt possui dois grupos de ajustes.

- Zona de proteção do alimentador (AL): cada alimentador é protegido por um relé (Ral, Ra2, Ra3 e Ra4) que atua sobre o religador associado.

- Zona de proteção do banco de capacitores (BC): protegida através do relé Rbc associado ao disjuntor $(11 \mathrm{H} 2)$.

O particionamento do SDF em módulos, de acordo com a classificação dos eventos na base de dados do sistema SCADA, facilita o desenvolvimento e a análise formal dos modelos, bem como a reutilização dos mesmos. Vale salientar que para as zonas de alimentadores, como os alimentadores da SE BBR possuem a mesma configuração, os mesmos serão representados por um único modelo RPC, sendo diferenciados somente pelas fichas coloridas. 


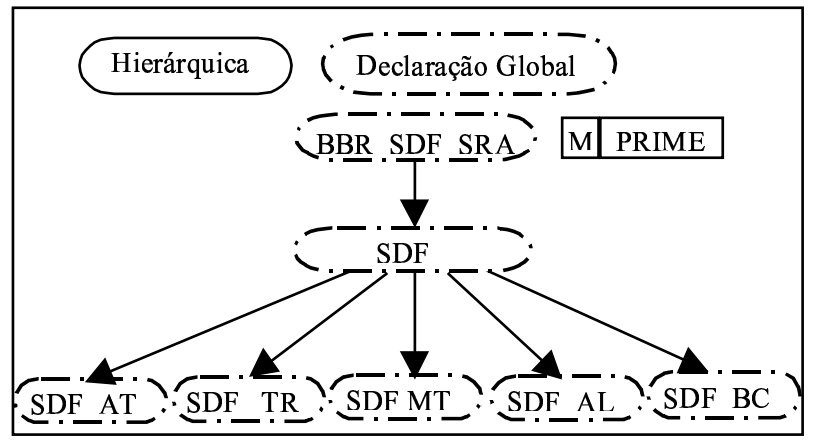

Figura 4: Página Hierárquica de uma Subestação de Distribuição.

\subsection{Hierarquia do Sistema de Diagnóstico de Faltas}

Na Figura 4 é apresentada a Página Hierárquica, a partir da qual são visualizadas e acessadas todas as páginas ou subredes que compõem o modelo do Sistema de Diagnóstico de Falta.

A página BBR_SDF_SRA, ilustrada na Figura 4, é a página principal do modelo SDF, a partir da qual visualizam-se os estados iniciais das zonas da subestação e os resultados de diagnósticos obtidos. Esta página contempla as transições de substituição SDF e SRA. A transição SDF modela o sistema de diagnóstico de falta e a transição SRA modela o sistema de restauração automática da SE BBR. O sistema SRA não está contemplado neste artigo. Na sub-página SDF são apresentadas as transições de substituição que modelam as zonas de proteção da SE BBR. As sub-páginas SDF_AT, SDF_TR, SDF_MT, SDF_AL, e SDF_BC modelam as respectivas zonas de proteção da SE BBR relacionadas aos respectivos vãos de Alta Tensão, Transformador, Média Tensão, Alimentadores e Banco de Capacitores.

A Declaração Global indicada na Figura 4 é a página onde são declarados os conjuntos de cores com os tipos de dados e as variáveis utilizadas na modelagem do SDF. A Figura 5 mostra a Página Declaração Global para o SDF da SE BBR. Nesta página o tipo Zona, por exemplo, define um conjunto de cores que pode assumir valores AT, TR, MT, AL e BC que representam, respectivamente, as zonas de proteção da subestação. Por exemplo, a variável z (var z) é do tipo Zona, podendo assumir os valores AT, TR, MT, AL e BC referentes às zonas de proteção.

\subsection{Módulo Principal do SDF}

O módulo principal do SDF para a SE BBR é composto pela rede principal apresentada na Figura 6 e pela sub-rede mos-
DECLARAÇÃO GLOBAL

color Zona = with AT|TR|MT|AL|BC ; (*Tipo Zona: representa as zonas da SE BBR*)

(*Tipo Proteção: representa dados dos relés de proteção*) color $\quad$ Protecao $=$ with Rel50A $|\operatorname{Rel} 50 \mathrm{~B}| \operatorname{Rel} 50 \mathrm{C}|\operatorname{Rel} 50 \mathrm{~N}| \operatorname{Rel} 51 \mathrm{~A}$

$\mid$ Rel51B|Rel51C|Rel51N |RelRvcc|RelTPvca|RelRwd (*Proteção da zona de AT *) Rta50A|Rta50B|Rta5 0C|Rta50N|Rta51A|Rta51B|Rta51C Rta51N|RtaRvcc|RtaTPvca|RtaRwd|Rtd50A|Rtd50B|Rtd50C|Rtd50N| Rtd51A|Rtd51B|Rtd51C|Rtd51N|Rtd87A|Rtd87B|Rtd87C|Rtd50G|RtdRv cc|RtdTPvca|RtdRwd|Rti63|Rti63A|Rti80|Rti26|Rti49 (* Proteção da zona de TR*)|Rtb50A|Rtb50B|Rtb50C|Rtb50 N| Rtb51A|Rtb51B |Rtb51C |Rtb51N|RtbRvcc|RtbTPvca|RtbRwd|Rdt50A|Rdt50B|Rdt50C|Rdt50N $\mid$ Rdt51A|Rdt51B|Rdt51C|Rdt51N|Rdt50ns|Rdt51ns|Rdt79|RdtRvcc|RdtT Pvca|RdtRwd(*Proteção da zona de $M T \quad *)|\operatorname{Ral50A}|$ Ral50B|Ral50C Ral50N|Ral51A $\mid$ Ral51B $\mid$ Ral51C $\mid$ Ral51N|Ral50ns $\mid$ Ral51 ns $\mid$ Ral79|RalRvcc $\mid$ RalTPvca|RalRwd(*Proteção da zona de AL*)|Rbc50A |Rbc50B| Rbc50C|Rbc50N|Rbc51A|Rbc51B|Rbc51C|Rbc51N|Rbc50ns|Rbc51ns |Rcb61|RbcRvcc|RbcTPvca|RbcRwd (*Proteção da zona de BC *);

(*Tipo Disjuntor: representa dados dos disjuntores e religadore $\mathrm{s}^{*}$ )

color Disjuntor $=$ with D12C5|D12D1|D12C5D1|D12C5D11T1|

D12C5D11D1|D12D1D11T1|D12D1D11D1|12C5T1D1|D12D1D1| D11T1|D11D1|D11T1D1|D21AL|D11H2|FD;

(*Tipo supervisao: representa dados de supervisão dos disjuntores*) color Supervisao $=$ with D12C5sf6a|D12C5s f6d|D12C5f27|D12C5ba |D12D1sf6a|D12D1sf6d|D12D1f27|D12D1ba|D11T1sf6a|D11T1sf6d |D11T1f27|D11T1ba|D11D1sf6a|D11D1sf6d|D11D1f27|D11D1ba|

D21ALsf6a|D21ALsf6d|D21ALf27|D21ALba|D11H2sf6a

|D11H2sf6d|D11H2f27|D11H2ba|Rtb50BF|Rdt50BF|Rbc50BF; (*Tipo Diagnóstico: representa o resultado do diagnóstico*) color Diagnostico $=$ product Sistema*Protecao*Disjuntor; (*Variáveis do Sistema*)

var z : Zona;

var $\mathrm{r}$ : Protecao;

var d : Disjuntor;

var s : Supervisao;

Figura 5: Declaração Global do SDF da SE BBR.

trada na Figura 7

Na Figura 6 é ilustrada a Página BBR_SDF_SRA, página principal do modelo, a partir da qual se tem uma visão geral da dinâmica do sistema elétrico da subestação. A marcação inicial do modelo SDF/SRA (1'AT, 1'TR, 1'MT, 1'AL, 1'BC), associada ao lugar Sistema, representa os estados iniciais das zonas de proteção da SE BBR. A Transição de Substituição SDF modela a ocorrência de uma falta e a atividade de diagnóstico de falta. Esta transição está associada à subrede SDF apresentada na Figura 7. O resultado do diagnóstico de falta é representado pela marcação final do lugar Resultado de Diagnóstico.

Na sub-rede SDF, Figura 7, o lugar Sistema apresenta a inscrição $\mathrm{P}_{I n}$, indicando que este lugar recebe as fichas enviadas pela rede principal SE_BBR mostrada na Figura 6. O lugar Resultado Diagnóstico com a inscrição $\mathrm{P}_{O u t}$ envia o resultado do diagnóstico para a rede principal SE_BBR. As transições de substituição SDF_AT, SDF_TR, SDF_MT, SDF_AL e SDF_BC estão associadas às sub-redes que modelam os sub-módulos das zonas de proteção. 


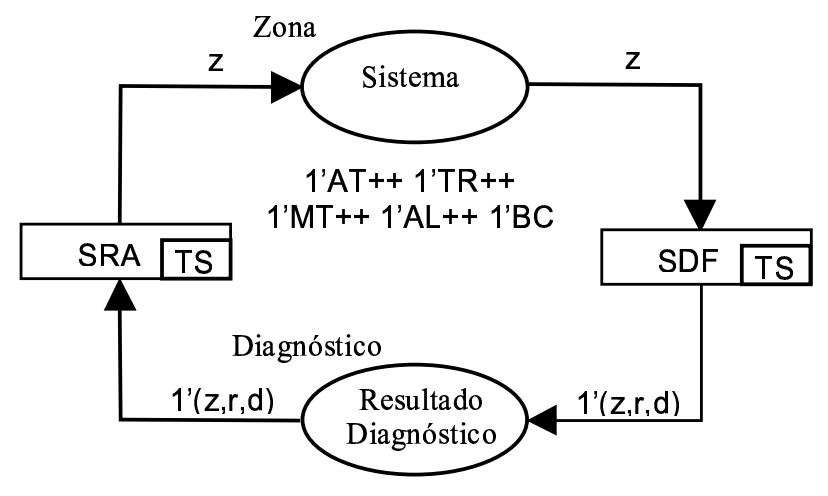

Figura 6: Modelo SDF/SRA Página Principal SE_BBR.

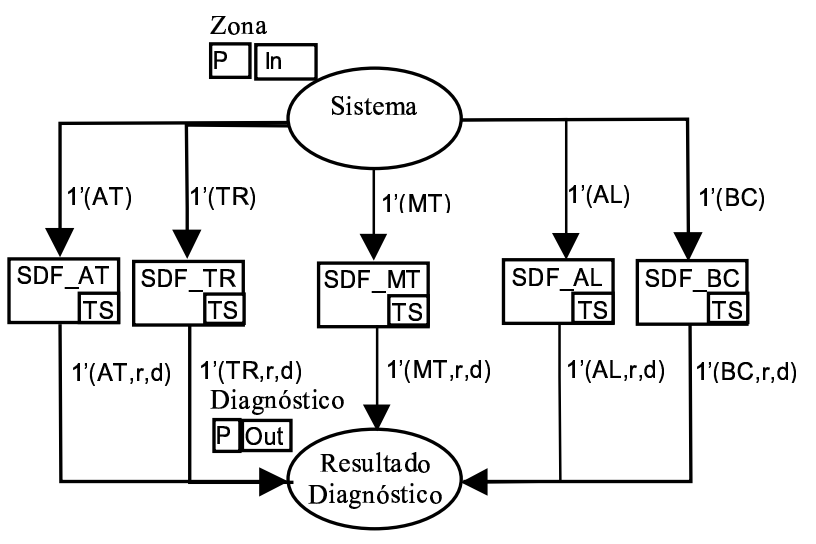

Figura 7: Sub-Página SDF.

\subsubsection{Modelo de Simulação do SDF para a Zona de Banco de Capacitores}

Nesta seção é apresentado o modelo do sistema de diagnóstico de falta desenvolvido para a zona de proteção do banco de capacitores (SDF_BC) da subestação Beberibe, conforme esquema de proteção apresentado na Figura 3. Através da simulação do modelo são apresentados a dinâmica do sistema e o diagnóstico de falta para uma ocorrência no banco de capacitores envolvendo atuação do relé do banco de capacitores Rbc e a falha do disjuntor do banco $11 \mathrm{H} 2$. A ocorrência simulada representa um caso real de uma falta por curto-circuito no banco de capacitores da subestação BBR envolvendo a fase A do banco com atuação da função de sobrecorrente instantânea do relé Rbc, a falha do disjuntor do banco de capacitores $11 \mathrm{H} 2$, e a atuação da função falha do disjuntor do relé Rbc sobre o disjuntor geral do barramento $11 \mathrm{~T} 1$.

De acordo com o esquema de proteção do banco de capacitores da SE Beberibe, quando ocorre uma falta no banco, o relé Rbc detecta a falta e envia um comando de abertura para o disjuntor do banco $11 \mathrm{H} 2$ que, por sua vez, interrompe a falta.

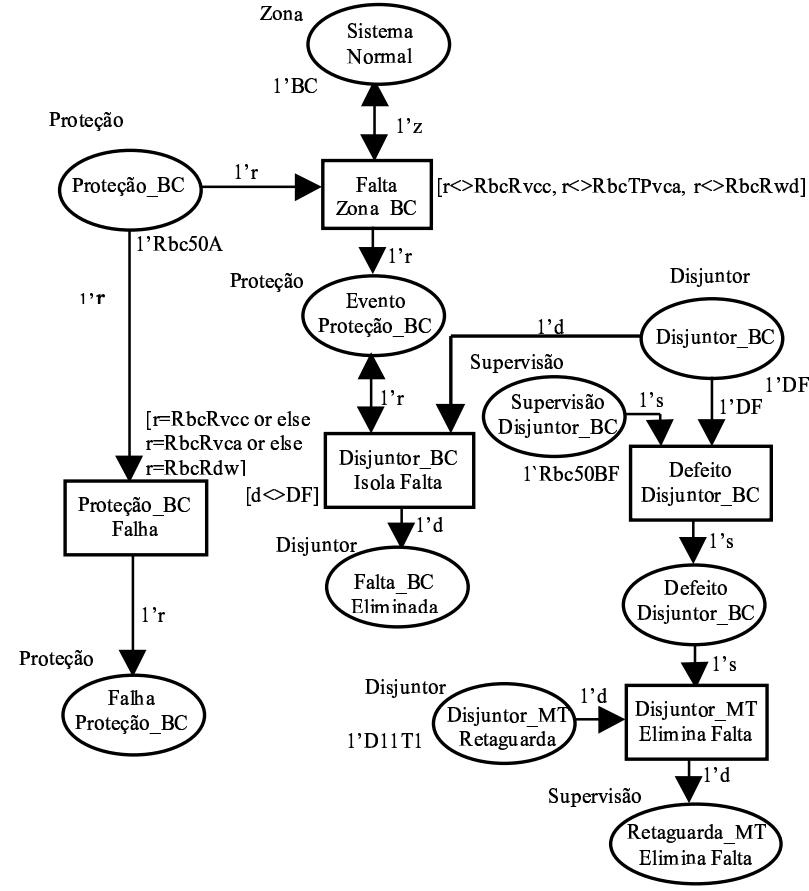

Figura 8: Modelo SDF_BC - Marcação Inicial.

Caso o disjuntor não elimine a falta no tempo predefinido, a função 'falha de disjuntor' envia um comando de abertura para o disjuntor de retaguarda, disjuntor geral do barramento $11 \mathrm{~T} 1 \mathrm{e} / \mathrm{ou}$ disjuntor de transferência 11D1 que interrompe a falta de acordo com a posição da chave 43T1b. O relé, além de atuar sobre os disjuntores, registra os eventos relacionados àquela ocorrência e transmite-os para o sistema SCADA.

O SCADA após coletar os eventos do relé, disponibiliza-os para o SDF que seleciona através da interface de entrada de dados as informações relevantes para o diagnóstico de falta e as codifica como marcação inicial na RPC-H. De posse da marcação inicial o módulo RPC relativo à zona de proteção do banco de capacitores é executado até encontrar uma marcação final, denominada de marcação morta na teoria de redes de Petri. A marcação final é então entregue à interface de saída do SDF e esta armazena e emite um relatório como resultado do diagnóstico de falta. Após o diagnóstico, o módulo SDF_BC retorna ao seu estado inicial, ou seja, sem fichas e pronto para receber uma nova marcação inicial quando da ocorrência de outro evento.

O modelo SDF_BC para a zona de proteção de banco de capacitor e a simulação do mesmo para a falta supracitada é apresentado na Figura 8. Neste modelo, fichas nos lugares de entrada Sistema Normal, Proteção_BC, Disjuntor_BC, Supervisão Disjuntor_BC e Disjuntor_MT Retarguarda representam as informações de ocorrências no sistema elétrico reportados pelo SCADA da SE BBR. As ocorrências 
Tabela 1: Marcação inicial do Modelo SDF_BC.

\begin{tabular}{|c|c|c|}
\hline $\begin{array}{ll}\text { Lugares } & \text { de } \\
\text { Entrada } & \text { de } \\
\text { Dados } & \\
\end{array}$ & $\begin{array}{l}\text { Marcação } \\
\text { Inicial }\end{array}$ & Significado do Código \\
\hline $\begin{array}{l}\text { Sistema nor- } \\
\text { mal }\end{array}$ & 1'BC & $\begin{array}{l}\text { A ficha 1'BC representa o } \\
\text { vão do BC. }\end{array}$ \\
\hline Proteção_BC & 1'Rbc50A & $\begin{array}{l}\text { A ficha 1'Rbc50A repre- } \\
\text { senta a atuação da função } \\
\text { de sobrecorrente instantânea } \\
\text { (50) e a fase que ocorreu a } \\
\text { falta (fase A). }\end{array}$ \\
\hline Disjuntor_BC & 1'DF & $\begin{array}{l}\text { A ficha 1'DF representa a } \\
\text { não abertura do disjuntor } \\
11 \mathrm{H} 2 \text { após a atuação da fun- } \\
\text { ção } 50 \text {. }\end{array}$ \\
\hline $\begin{array}{l}\text { Supervisão } \\
\text { Disjun- } \\
\text { tor_BC }\end{array}$ & $1^{\circ} \mathrm{Rbc50BF}$ & $\begin{array}{l}\text { A ficha } 1 \text { 'Rbc50bf repre- } \\
\text { senta a atuação função falha } \\
\text { de disjuntor (50bf) do relé } \\
\text { Rbc, após a falha do disjun- } \\
\text { tor } 11 \mathrm{H} 2 \text {. }\end{array}$ \\
\hline $\begin{array}{l}\text { Disjuntor_MT } \\
\text { Retaguarda }\end{array}$ & 1'D11T1 & $\begin{array}{l}\text { As fichas 1' D11T1 repre- } \\
\text { senta o disjuntor principal do } \\
\text { barramento abertos pela fun- } \\
\text { ção 50BF. }\end{array}$ \\
\hline
\end{tabular}

das transições representam as mudanças no estado do sistema, e fichas nos lugares Evento Proteção_BC, Falha Proteção_BC, Falta_BC Eliminada, Defeito Disjuntor_BC e, Retarguarda_MT Elimina Falta modelam o diagnóstico. Na Tabela 1 é apresentada a marcação inicial para a referida ocorrência.

A Figura 9 apresenta a nova marcação da RPC após o disparo da transição Disjuntor_MT Elimina Falta que corresponde à atuação da função falha do disjuntor do relé do banco sobre os disjuntores principais. Note que na Figura 9 é apresentada a marcação final especificada na Tabela 2.

Com base na marcação final da Tabela 2, obtém-se o seguinte diagnóstico de falta:

- falta na fase A do vão de banco de capacitores;

- função de sobrecorrente instantânea (50) do relé atuou;

- disjuntor do banco 11H1 falhou;

- atuação da função falha de disjuntor do relé do banco (50BF);

- abertura do disjuntor geral do barramento de $13,8 \mathrm{kV}$ (11T1).

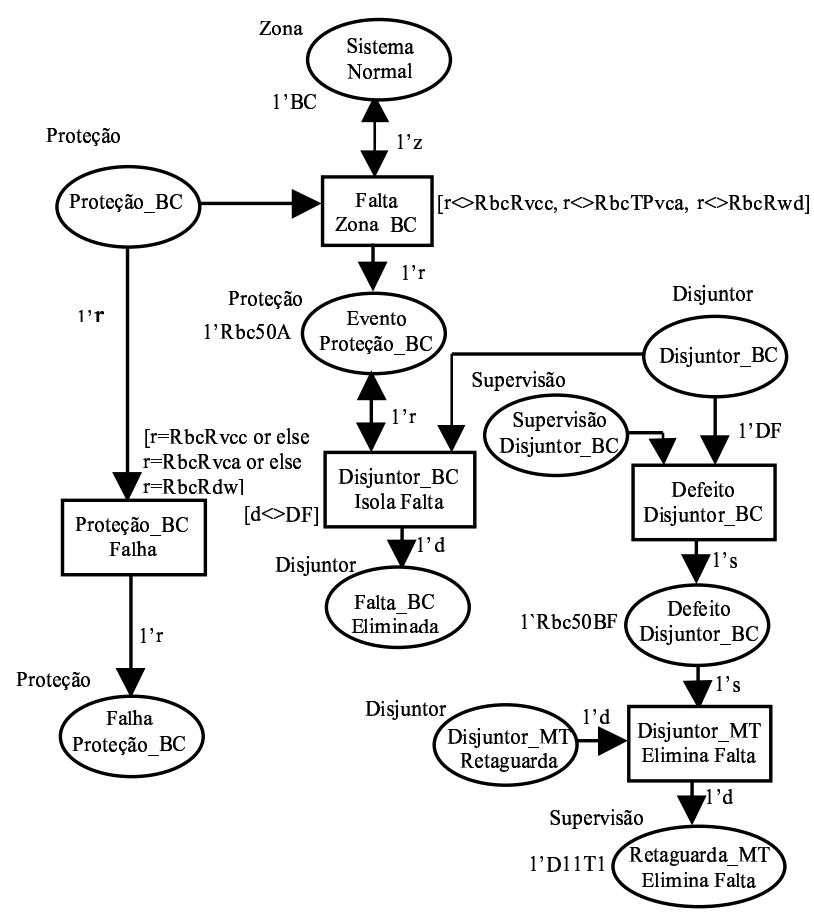

Figura 9: Modelo SDF_BC - Abertura dos Disjuntores de Retaguarda pela Função 50BF do relé Rbc.

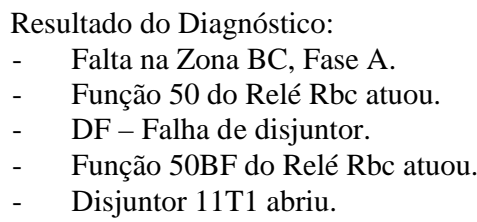

Figura 10: Resultado do Diagnóstico para Falta na Zona BC.

Para esta ocorrência que trata de uma falta com falha de disjunção, o SDF_BC filtra os dados importantes para o diagnóstico da falta, e emite o seguinte relatório com o resultado do diagnóstico da falta, para auxíliar o profissional da operação na tomada de decisão rápida e segura.

Como pode ser observado, no relatório de diagnóstico da falta emitido pelo SDF constam: a zona de proteção onde ocorreu a falta, o relé e as funções de proteção que atuaram, a fase na qual ocorreu a falta, o disjuntor que falhou e o disjuntor que interrompeu a falta.

Inúmeros testes de ocorrências reais nos vários vãos da $\mathrm{SE}$ BBR foram realizados, Os testes executados apresentaram diagnósticos corretos em todas as faltas e falhas simuladas, com um tempo de execução de frações de segundos após a ocorrência, visto que o número de marcações alcançadas em cada execução é de no máximo uma dezena de marcações. 
Tabela 2: Marcação Final para Falta na Zona_BC

\begin{tabular}{|c|c|c|}
\hline Lugar & $\begin{array}{l}\text { Marcação } \\
\text { Final }\end{array}$ & Decodificação \\
\hline $\begin{array}{l}\text { Sistema } \\
\text { Normal }\end{array}$ & 1'BC & $\begin{array}{l}\text { BC - Vão do banco de } \\
\text { capacitores }\end{array}$ \\
\hline $\begin{array}{l}\text { Evento Prote- } \\
\text { ção_BC }\end{array}$ & 1'Rbc50A & $\begin{array}{l}\text { Rbc - Relé do banco de } \\
\text { capacitores } \\
50 \text { - Função de sobrecor- } \\
\text { rente instantânea do relé } \\
\text { Rbc que atuou no mo- } \\
\text { mento da falta } \\
\text { A - Fase na qual ocorreu } \\
\text { a falta }\end{array}$ \\
\hline $\begin{array}{l}\text { Defeito Dis- } \\
\text { juntor_BC }\end{array}$ & 1'Rbc50Bम & $\begin{array}{l}\text { Rbc - Relé do banco de } \\
\text { capacitores } \\
\text { 50BF - Função falha de } \\
\text { disjuntor }\end{array}$ \\
\hline $\begin{array}{l}\text { Retaguarda_MT } \\
\text { Elimina Falta }\end{array}$ & 1'11T1 & $\begin{array}{l}\text { 11T1 - Disjuntor geral } \\
\text { do barramento de média } \\
\text { tensão }\end{array}$ \\
\hline
\end{tabular}

\section{CONCLUSÃo}

Neste trabalho foi apresentado um método para sistemas de diagnóstico de faltas que utiliza uma abordagem distribuída baseada no particionamento da subestação por zona de proteção, caracterizando um sistema de diagnóstico localmente distribuído. O método confere à ferramenta modularidade, expansibilidade e manutenabilidade. Para implementação do SDF foram utilizadas as redes de Petri Coloridas Hierárquicas, adequadas ao método proposto. $\mathrm{Na}$ implementação utilizou-se o Design CPN que trata-se de uma ferramenta de modelagem, análise e validação de RPC-H, sendo executada em plataforma Linux.

Dentre os vários benefícios, o SDF proposto permite: informar as causas da ocorrência na subestação, de forma rápida e precisa; tornar a tarefa de diagnóstico de falta menos estressante e menos complexa para os operadores; reduzir o custo operacional de análise de ocorrências; e, contribuir na melhoria dos índices de confiabilidade da empresa.

Baseados nos testes realizados a partir de ocorrência de faltas nas várias zonas de proteção da subestação, com obtenção de resultados satisfatórios, conclui-se que esta técnica é adequada para modelagem, análise e validação de sistemas de diagnóstico de falta. Constata-se desta forma que a linha de pesquisa que utiliza RPC-H apresenta-se como uma potente ferramenta de auxílio à operação em tempo real.

Atualmente está em desenvolvimento um sistema de diagnóstico de falta baseado em RPC-H que integra as várias subestações de um sistema elétrico de potência e em estudo um sistema de restauração automático com base no resultado do diagnóstico.

\section{REFERÊNCIAS}

Brittes, J. L. P. (Set./Out. 1998). Função de Apoio ao Operador no Diagnóstico de Atuação do Sistema de Controle e Proteção da Subestação. VI STPC - Seminário Técnico de Proteção e Controle. pp. 1-7.

Chen, W. H., Liu, C.W., Tsai, M. S. (April 2000). OnLine Fault Diagnosis of Distribution Substations Using Hybrid Cause-Effect Network and Fuzzy Rule-Based Method. IEEE Transactions on Power Delivery. v. 15, n. 2, pp. 710-717.

Infran, A. H. F. et al. (Ago./Set. 1995). Processamento de Alarmes em Unidades Geradoras de Grande Porte Usando Redes Neurais. V Seminário Técnico de Proteção e Controle. pp. 145-152.

Jansen, K. (1992). Colored Petri Nets - Basic Concepts, Vol.1. Monograph in Theoretical Computer Science. Springer-Verlag.

Jansen, K. (1994). Colored Petri Nets - Analysis Methods, Vol.2. Monograph in Theoretical Computer Science. Springer-Verlag.

Jansen, K. (1997). Colored Petri Nets - Practical Use. Vol.3. Monograph in Theoretical Computer Science. Springer-Verlag.

Lee, H. J., Park, D. Y., Ahn, B. S., Park, Y.M., Park, J. K., Venkata, S.S..(April 2000). A Fuzzy Expert System for the Integrated Fault Diagnosis. IEEE Transactions on Power Delivery. v. 15, n. 2, pp. 833-838.

Lo, K.L., Ng. H. S. and Trecat. J. (May. 1997). Power Systems Fault Diagnosis Using Petri Nets. IEE Proceedings on Generation, Transmission and Distribution. Vol.144, No. 3, pp. 231-236.

Murata. T. (April 1989). Petri Nets: properties, analysis and applications. Proceedings of the IEEE. Invited paper. Vol. 77, No. 4, pp. 541-580.

Sampaio, R. F. (Junho 2002). Sistema de Diagnóstico de Faltas para Subestações Baseado em Redes de Petri Coloridas. Dissertação de Mestrado. Universidade Federal do Ceará.

Sampaio, R. F., Barroso, G. C., and Leão, R.P.S. (Sept./2002). System of Fault Diagnosis for Electric Power System Based on Colored Petri Nets, I Brazilian Meeting on Petri Nets (BMPN). Natal (RN), Brazil. Vol.1, pp.1-6. 
Sampaio, R. F., Barroso, G. C. and Leão, R. P. S. (February 2003). An Advanced Function for the Supervisory System of an Electrical Distribution Substation: An Application Using Colored Petri Nets. International Conference on Modeling and Simulation (IASTED) (MS003). California, USA. Vol.1; pp.192-197.

Silva, V. N. A. L., Souza, G. N. F., Zaverucha, G. (Ago./Set. 1995). Diagnóstico em Sistema de Potência utilizando Sistemas Especialistas. V Seminário Técnico de Proteção e Controle. Vol.1, pp. 297-303.

Zhu, Jun, Lubkeman, David L. (May 1997). ObjectedOriented Development of Software Systems for Power System Simulations. IEEE Transactions on Power Systems. v. 12, n. 2, pp. 1002-1007.

Vale, Maria H. M., Vale, M. I. M., Parma, G. G., Faria, V. R. (Dez./2003). Sistema de Tratamento de Alarmes Aplicado ao Sistema da CEMIG. EletroEvolução Sistemas de Potência. CIGRE-Brasil. n.33. p.63-69, 2003.

Yang, C.L., Yokoyama, A. (1995). Fault section Estimation of Power System using Color time Petri Nets. Electric Engineering in Japan. v. 115, n. 2, pp. 89-101. 